Ablation of polytetrafluoroethylene (Teflon) with femtosecond UV excimer laser pulses

S. Küper and M. Stuke

Citation: 54, 4 (1989); doi: 10.1063/1.100831

View online: $\mathrm{http}: / / \mathrm{dx}$.doi.org/10.1063/1.100831

View Table of Contents: http://aip.scitation.org/toc/apl/54/1

Published by the American Institute of Physics

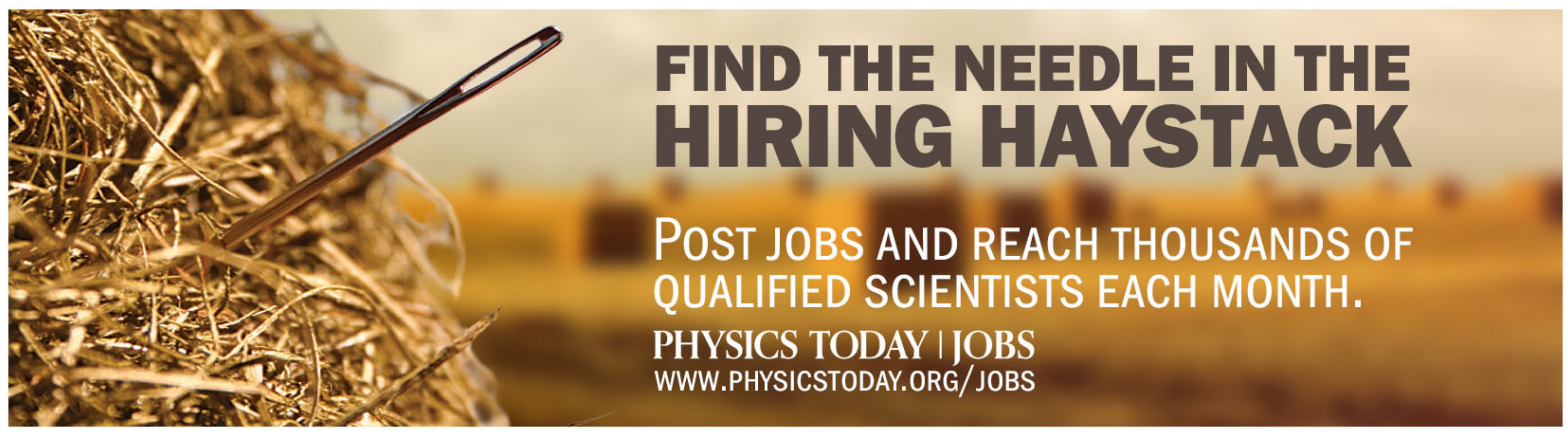




\title{
Ablation of polytetrafluoroethylene (Telion) with femtosecond UV excimer laser pulses
}

\author{
S. Küper and $M$. Stuke \\ Max-Planck-Institut fü Biophysikalische Chemie, Dept. Laserphysik, P. 0. 2841, D-3400 Götingen, \\ Federal Republic of Germany \\ (Received 15 February 1988; accepted for publication 23 October 1988)

\begin{abstract}
excimer laser pulses at $248 \mathrm{~nm}$ are reported for the first time. In contrast to standard excimer laser pulses, these ultrashort pulses ablate Teflon with good edge quality and no signs of thermal damage for fuences down to $0.5 \mathrm{~J} / \mathrm{cm}^{2}$ with removal rates on the order of $1 \mu \mathrm{m}$ per pulse.
\end{abstract} \\ Experiments on the ablation of undoped polytetrafluoroethylene (Teflon) with 300 fs UV
}

Polymer surfaces can be structured by exposure to intense UV laser light. ${ }^{1,2}$ A wide variety of polymers with different penetration depths for UV laser light and different chemical constitution shows for certain fuence ranges high removal rates of material on the orcer of micrometers per pulse, along with little thermal damage to the edges. Thus the surfaces of many polymers can be palterned with a resolution down to less than $1 \mathrm{~km}$. $^{3}$

Some polymers, such as undoped polytetrafuorocthylene (Tefion) however, are not accessible to clean ablation with stankard excimer laser pulses at 308,248 , or $193 \mathrm{~nm}$. Under these conditions the material is photochemically and thermally degraded ${ }^{4}$ and, if at all, rather distupted than etched by ablation. Recently, it has been shown that ultrashort excimer laser pulses ${ }^{5,6}$ offer advantages for the structuring of polymer surfaces, and are a valuable tool for mechanistic studies of the ablation process. ${ }^{7,8}$ Furthermore, new materials have become accessible for ablation with 248 nm excimer laser pulses. In the following, we stall describe ablation cxperiments with Tefon at $248 \mathrm{~nm}$ for excimer laser pulse durations of $16 \mathrm{~ns}$ and $300 \mathrm{fs}$.

Materials: 4-mm-thick commercial Teflon sheets $\left(\alpha_{248}=158 \mathrm{~cm}^{-1},{ }^{10}\right.$ density $\left.=2.15 \mathrm{~g} / \mathrm{cm}^{3}\right)$ were used for the determination of etch depth as a function of fluence. Before irradiation, the surface of the samples was polished and cleaned with methanol.

procedure: The light sources and the experimertal setup have been described in detail before. ${ }^{7}$ The repetition rate of the laser pulses was kept at $1 \mathrm{~Hz}$ in order to exclude effects arising from heating of the sample. The laser beam was defined by a metal mask, focused by a spherical quartz lens $(f=200 \mathrm{~mm})$ and directed at the sample. For each given fiuence several exposures at varying numbers of pulses were made and the etch depth of each hole was measured with a Dektak 11 A stylus profilometer. Etch rates were obtained by inear regresssion from the slope of a plot of the etch depth versus the number of pulses (compare Fig. 3 of Ref. 7 ), which showed excellent linearity. All irradiations were conducted in air atmosphere.

Figure 1 shows the scanning electron micrograph (SEM) of a Tefion sample which had been exposed to 50 pulses of $2 J / \mathrm{cm}^{2}$ at $248 \mathrm{~nm}$ with a pulse duration of $16 \mathrm{~ns}$ FWHM (full widh at hall maximum). It is obvious that the observed result cannot be termed ablation, as well as exact etch rates cannot be obtained due to the surface morphology, which prevails the same, also for higher fiuences at the same pulse duration. In contrast, 300 fs pulses at the same wavelength smoothly ablate Tefion at fuences down $100.5 \mathrm{~J} / \mathrm{cm}^{2}$. Figure 2 shows a typical sample which had been subject to 240 pulses of $1 \mathrm{~J} / \mathrm{cm}^{2}$ at $248 \mathrm{~nm}$ with a pulse duration of 300 fs. The etch pit is sharply defined. The unevenness of the bottom of the etch hole is due to inhomogeneities in the bear: profile. The ablated surftce exhibits a roughness on the order of $1 \mu \mathrm{m}$ and there is practically no solid debris at the edges of the hole. Figure 3 shows the measured etch rate of Tellon for pulses of $300 \mathrm{fs}$ duration at $248 \mathrm{~mm}$ plotted versus the fuence. Below $300 \mathrm{~mJ} / \mathrm{cm}^{2}$ virtually no ablation takes place. For higher fuences, a typical etch curve is observed, which levels of into a saturation plateau for fuences above $1 \mathrm{~J}$ $\mathrm{cm}^{2}$. A careful measurement of the etch depth versus the number of incident pulses shows a linear rise from the first pulse on. This means that in the covered fuence range, and for $248 \mathrm{~nm}$, Tefon, in contrast to other materials, does not show incubation behavior.,

With an absorption coefficient of $158 \mathrm{~cm}^{-1}$ (Ref. 10) Tefon is an equally weak absorber as PMMA with 102 $\mathrm{cm}^{-1}$ (Ref. 7). PMMA exhibits a rapidly growing UV absorption under excimer laser irradiation due to the formation of stronger absorbing unsaturated species, and, at moderate fluences at $248 \mathrm{~nm}$, starts ablation only after the material has been sensitized by several incubation pulses. ${ }^{7,9}$

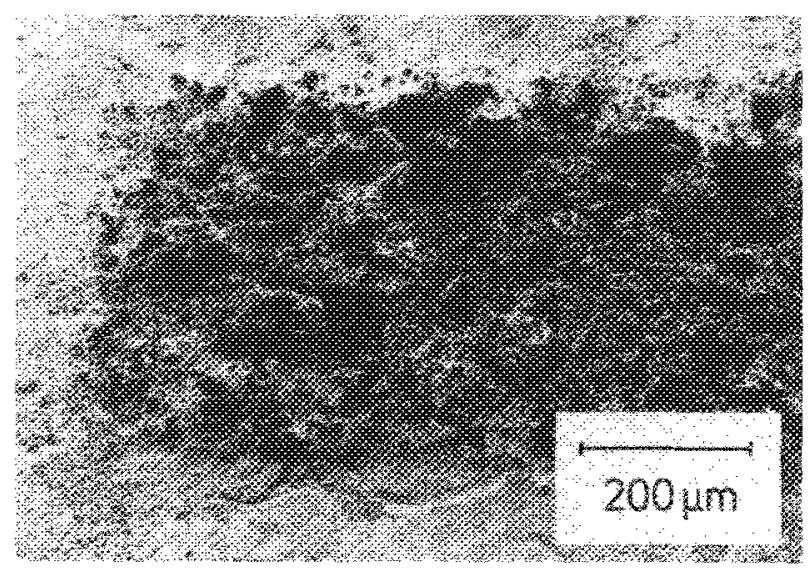

FIG. 1. SEM pirotograph of Teflon surface exposed to 50 pulses of $23 / \mathrm{cm}^{2}$ and $16 \mathrm{~ns}$ FWHM at $248 \mathrm{~nm}$. The material is severely degraded (see Ref. 4) and rather disrupted than etched by ablation. 


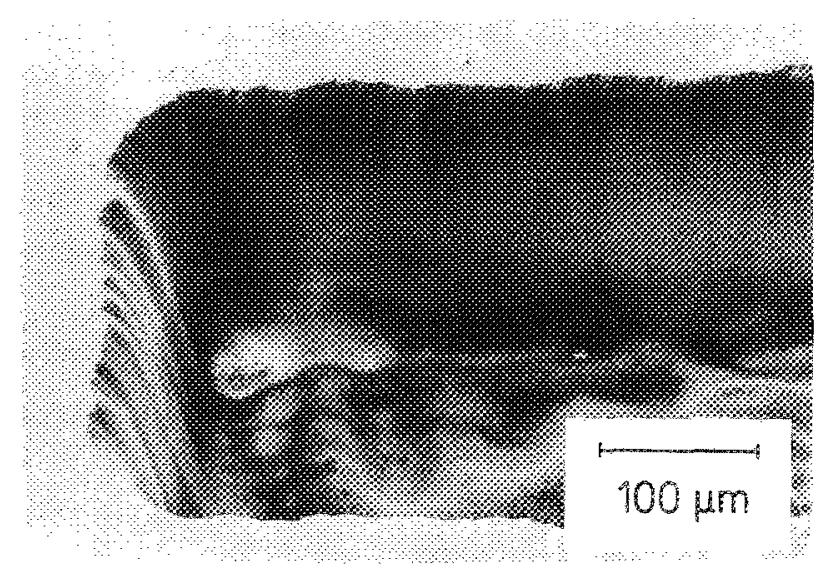

FIG. 2. SEM photograph of Tefon surface subject to $240 \mathrm{pulses}$ of $1 \mathrm{~J} / \mathrm{cm}^{2}$ and 300 fs pulse duration at 248 nm. The material is sinoothly ablated with no thermal damage to the edges.

This means that material modification resulting from photon absorption does accumulate over consecutive pulses before ablation starts. In the case of Teflon however, there is no evidence in the UV and infrared spectra for a growing UV absorption or formation of unsaturated species, ${ }^{10}$ also the number of incubation pulses $N_{t}=0$ (compare Fig. 3 of Ref. 7). At the same time, Teflon has no favored sites for bond scission (weak bonds), but a bond energy of at lcast $406 \mathrm{~kJ} /$ mole" has to be overcome, whereas in PMMA chain scission is facilirated by a dissociation energy of oniy $259 \mathrm{~kJ} /$ mole ${ }^{\prime}$ for the weakest bond. Considering energy losses due to various relaxation processes, the probability that suffcient energy for scission is accumulated within one chemical bond decreases with a rising bond energy and increases with the rate of coupling the energy into the material. The effects of a constant, weak UV absorption along with high bond energies make Teflon relatively inert towards intense UV radiation. Their different absorption coefficients and densities taken into account, the photon load II per monomer unit and unit time under ns excimer laser irradiation for Teflon and PMMA is about the same ( $\left\|_{\text {Tethon }}=0.85 \times\right\|_{\text {PMMA }}$ ). However, more than 30000 pulses of 16 ns duration and 120 $\mathrm{mJ} / \mathrm{cm}^{2}$ do not induce any observable change in the infrared and UV spectra of a $12.5 \mu \mathrm{m}$ thin Teflon film, ${ }^{10}$ while under the same conditions PMMA undergoes severe photochemical degradation. This shows that Teflon requires a higher photon load before suffering damage to its chemical structure. With extremely high light intensities, ultrashort excimer laser pulses induce nonimear absorption ${ }^{7.12}$ and therefore achieve a high rate of coupling energy into materials, absorbing weakly under low intensity conditions. Thus the various relaxation processes can be overcome in favor of bond scission, and ablation becomes possible. In the case of fs ablation, the observed threshold fluence for Teflon is three times higher than for PMMA under equal conditions, a fact, which is in agreement with its constant, weak absorption and higher bond energies, compared to PMMA.

In an extension ${ }^{7}$ of the model given in Ref. 9, we have explained the leveling of the etch curve at high fuences by the dynamic behavior of the material's absorption coefficient under excimer laser irradiation. Our model takes into

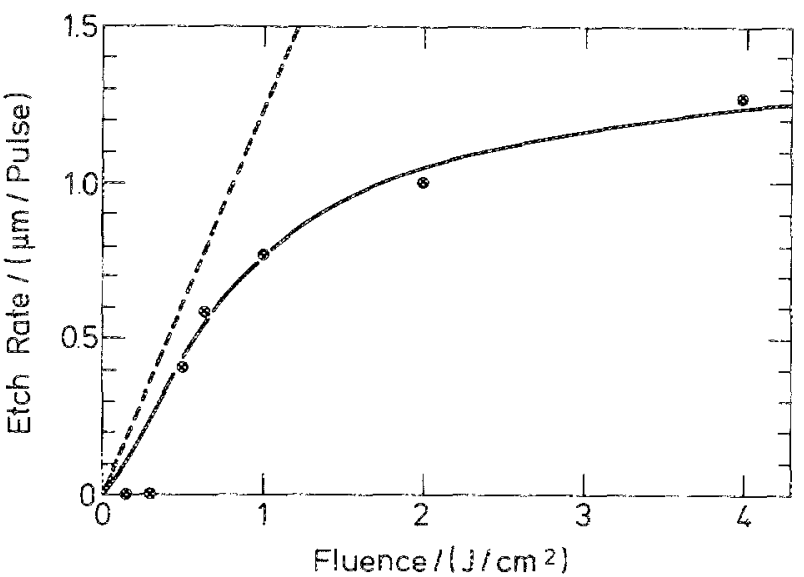

FIG. 3. Etch rate v exciner laser fuence for the ablation of Tefon at 248 $\mathrm{nm}$ with ultrashort excimer laser pulses of $300 \mathrm{f}$ duration. Solid circles: expermont. Solid line: calculated etch curve, faking into account an intensity-dependent absorption coefficient (see Ref. 7). Broken line: etch curve calculated irrespective of multiphoron effects (sce Ref. 9 ).

account a dependency of the absorption coeficient on the intensity of the incident laser light and, depending on the material, on the absorbed irradiation dose. The effective $a b$ sorption coeficient $\alpha^{\prime}$ of material under UV irradiation can be expressed by

$$
\alpha^{p}=\alpha+(\beta-\alpha) n_{\mathrm{inc}} / n+\delta I_{(t, x)},
$$

where $\alpha$ is the low intensity absorption coefficient of virgin material, $\beta$ of an incubation site formed by irradiation, $n_{\mathrm{inc}}$ is the number density of incubation sites, and $n$ is the initial number density of monomer units. The second term $(\beta-a) n_{\text {in: }} / n$ describes the change in absorption due to the irradiation dose, the third term $\delta I_{(t, x)}$ the contribution of two-photon absorption to the effective absorption coefficient $\alpha^{\prime}$. The latter increases $\alpha^{\prime}$ significantly only for incident UV light intensities in the range of $100 \mathrm{GW} / \mathrm{cm}^{2}$ or higher, which are achieved by ultrashort excimer laser pulses. ${ }^{5,6}$ The two-photon absorption coefficient $\delta$ is assumed to be independent of the local light intersity $I_{(s, x)}$, which means that multiphoton absorption of higher orders is not taken into account. The above equation is identical to Eqs. (1) and (2) of Ref. 7 with $\delta=\alpha_{\text {eff }} \gamma$. This scaling of the multiphoton absorption is done in order to allow for materials with vanishing low intensity absorption and to allow an easier comparison with the experimental results obtained by others. ${ }^{2}$ For each interval of the laser pulse the absorption coeficient $\alpha^{\prime}$ is calculated for every layer in the material. For the case of $\beta=\alpha$ and $\delta=0$ our model includes the approach of Ref. 9 .

Since, in the case of Tefion, UV spectra provide no evidence for an increased absorption of the irradiated material, $\beta$ is set equal to $\alpha$ for our calculations here, while a value of $\delta \neq 0$ is essential for the results. The calculated curve is, at higher fuences, very sensitive to the value of $\delta$, and the best fit to our experiments is obtained with a value of $\delta=4.65 \times 10^{-9} \mathrm{~cm} / \mathrm{W}\left(\mathrm{PMMA}^{7}: \delta=1.0 \times 10^{-8} \mathrm{~cm} / \mathrm{W}\right)$, compare to Ref. 12. The high light intensities of ultrashort pulses will exceed by far any reasonable value ${ }^{9}$ given for the fux threshold $\Pi_{T}$, and therefore the curve fit for fs pulses is not sensitive to this parameter. The ablation threshold $\rho_{T}$ is 
0.78 photons/monomer unit (PMMA ${ }^{7}: \rho_{T}=0.48$ photons/ monomer unit). In its present state of development our mod$\mathrm{e}$ is in quantitative agreement with the experiment for fuences higher than $500 \mathrm{~mJ} / \mathrm{cm}^{2}$, but does not explain the observed threshold fuence. A further extended model, in which for Teflon the material nodification, induced by the absorbed photons, does not accumulate over consecutive pulses in order to reach the ablation threshold $\rho_{r}$, also shows a threshold behavior for is ablation. Further work along this line is in progress.

In conclusion, we have shown that weak UV absorbers with highly stable molecular structure, such as Tefion, which were heretofore not accessible for ablation, can be clearly etched with ultrashor excimer laser pulses of 248 nm for the first time. Taking advantage of multiphoton absorption, fs excimer laser pulses can create their own absorption. The reduced penetration depth of the laser light limits the etch rate at high fluences, but makes new materials accessible to ciean ablation.

We would like to thank S. Szatmári and F. Schäfer for giving us the opportunity to use their source of 300 fs excimer laser radiation at $248 \mathrm{~nm}$. Technical assistance by $\mathrm{K}$. Müler and W. Sauermann and financial support by Sonder- forschungsbereich 93 ("Photochemie mit Lasern", Teliprojekt $\mathrm{C} 2, \mathrm{C} 15$ ) and Bundesministerium fur Forschung und Technologie (No. 13N5398/7) are gratefully acknowledged.

'Y. Kawamura, K. Toycda, and S. Namba, Appl. Phys. Lett. 40,374 (1982); J. Appl. Phys. 53, 6489 (1982).

'R. Srinivasan and V. Mayne-Banton, Appl. Phys. Lett. 41, 576 (1982).

${ }^{3}$ M. Rothsenild and D. J. Ehrich, 3. Vac. Sei. Technol. B 5, 389 (1987).

${ }^{-} \mathrm{M}$. Stuke and Y. Zhang, in Proceedings of the Symposium on Laser Processes for Microelectronic Applications, edited by J. J. Ritsko, D. J. Ehrhich, and M. Kashiwagi (Electrochemical Society, Pennington, NJ, 1988), p. 70.

'S. Szatmári, F. P. Schäfer, E. Müller-Horsche, and W. Mückenheim, Opt. Commun. 63, 305 (1987).

'J. Glownia, J. Misewich, and P. P. Sorokin, J. Opt. Soc. B 4, 1061 (1987).

'S. Küper and M. Stuke, Appl. Phys. B 44, 199 (1987).

${ }^{8}$ R. Srinivasan, E. Sutcliffe, and B. Braren, Appl. Phys. Lett. 51, 1285 (1987).

${ }^{3} \mathrm{E}$. Sutcliffe and R. Srinivasan, J. Appl. Phys. 60, 3315 (1986)

"G. Küper and M. Stuke (unpublished cesults).

"I. Mita, in Áspects of Degradation and Stabilization of Polymers, edited by H. II. G. Jallinek (Elsevier, Amsterdam, Oxford, New York, 1978), p. 254.

${ }^{12}$ A. J. Taylor, R. B. Gibson, and J. P. Roberts, in Technical Digest, Conference on Lasers and Electro-Optics (Optical Society of America, Washington, $\mathrm{BC}, 1988)$, paper WD 1 . 